DOI: https://doi.org/10.14311/TPFM.2022.025

\title{
MAXIMUM MODULUS ESTIMATES FOR THE LINEAR STEADY STOKES EQUATIONS IN DOMAINS OF $\mathbb{R}^{n}(n \geq 2)$
}

\section{W. Varnhorn}

Department of Mathematics, Faculty Mathematics and Natural Sciences, University of Kassel, Heinrich-Plett-Str. 40, 34125 Kassel, Germany

\begin{abstract}
A maximum modulus estimate for the Stokes system in bounded and unbounded domains of $\mathbb{R}^{n}(n \geq 2)$ is established via methods of hydrodynamical potential theory. Using a suitable potential ansatz in form of hydrodynamical layer potentials the method is based on the unique solvability of the resulting boundary integral equations' system of Fredholm type. In addition, in case of bounded domains, a projection onto the normal field on the boundary is required.
\end{abstract}

Keywords: Stokes equations, maximum modulus estimate, potential theory.

\section{Introduction}

The classical maximum principle states that a smooth non-constant function $u$ being harmonic in a bounded domain $\Omega \subset \mathbb{R}^{n}$ takes its maximum value on the boundary $\partial \Omega$, i. e.

$$
\max _{x \in \bar{\Omega}} u(x) \leq \max _{y \in \partial \Omega} u(y)
$$

Here $\bar{\Omega}:=\Omega \cup \partial \Omega$ is the closure of $\Omega$.

This principle extends also to solutions of more general elliptic equations with regular coefficients. It is not true, however, for solutions of higher order equations or of elliptic systems. In the present paper, instead of the Laplace equation $-\Delta u=0$ in $\Omega$, we consider the Stokes system

$$
\begin{aligned}
-\Delta v+\operatorname{grad} p & =0 & & \text { in } \Omega, \\
\operatorname{div} v & =0 & & \text { in } \Omega, \\
v & =b & & \text { on } \partial \Omega .
\end{aligned}
$$

This system is well-known in hydrodynamics: Here the vector $v=\left(v_{1}, v_{2}, \ldots, v_{n}\right)$ is the velocity field and $p$ some kinematic pressure function of a linearized viscous incompressible fluid flow defined in a domain $\Omega \subset \mathbb{R}^{n}$ with a smooth boundary $\partial \Omega$. The function $b \in C^{0}(\partial \Omega)$ is a given continuous boundary value, satisfying in case of bounded $\Omega$ the necessary compatibility condition

$$
\int_{\partial \Omega} b(y) \cdot N(y) \mathrm{d} o=0,
$$

where $N=N(y)$ denotes the exterior (with respect to $\Omega$ ) unit normal vector in $y \in \partial \Omega$.

In the above case, for the real-valued function $|v|$ there is no maximum principle. To see this, consider in the 2-d unit ball

$$
\Omega:=B_{1}(0):=\left\{x=\left(x_{1}, x_{2}\right) \in \mathbb{R}^{2}:|x|<1\right\} \subset \mathbb{R}^{2}
$$

the functions

$$
v_{1}\left(x_{1}, x_{2}\right):=x_{2}^{2}-1, \quad v_{2}\left(x_{1}, x_{2}\right):=x_{1}^{2}-1, \quad p\left(x_{1}, x_{2}\right):=2\left(x_{1}+x_{2}\right) .
$$




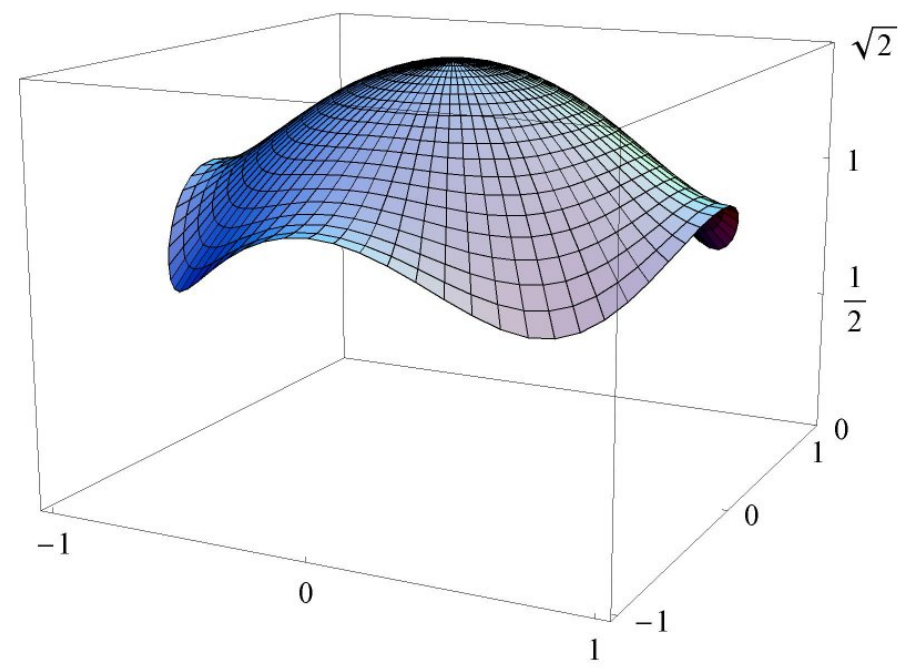

These functions represent a solution of the Stokes system in the unit ball $\Omega$, and it holds

$$
\frac{1}{2} \sqrt{2} \leq|v(x)|=\sqrt{x_{1}^{4}+x_{2}^{4}} \leq 1 \text { on } \partial \Omega,
$$

while $|v(0,0)|=\sqrt{2}$. Hence there is no maximum principle for the function $|v|$.

Nevertheless, a so-called maximum modulus estimate might be valid in this case. This means to prove the existence of some constant $c=c_{\Omega} \geq 1$ depending only on $\Omega$ with

$$
\max _{x \in \bar{\Omega}}|v(x)| \leq c \max _{y \in \partial \Omega}|v(y)|
$$

for all solutions of the Stokes equations.

\section{Main Results}

Our main result concerning the Stokes equations in bounded domains is now stated in

Theorem 2.1: Let $\Omega \subset \mathbb{R}^{n}(n \geq 2)$ be a bounded domain with a compact boundary $\partial \Omega \in C^{1, \alpha}$ $(\alpha>0)$. Let $b \in C^{0}(\partial \Omega)$ be a given boundary value satisfying (1). Then there is a constant $c=c_{\Omega}>0$ such that the solution $v$ of the Stokes system satisfies a maximum modulus estimate of the type (2).

We can prove this theorem using the method of boundary integral equations as follows. First we choose for the solution $v$ a representation in form of a suitable layer potential with an unknown source density $\psi$ :

$$
v(x):=D_{n} \psi(x):=\int_{\partial \Omega} D_{n}(x, y) \psi(y) d o_{y}, \quad x \in \Omega .
$$

Approaching the boundary, this representation leads to a system

$$
b(x)=A_{n} \psi(x), \quad x \in \partial \Omega
$$

of boundary integral equations, where $A_{n}: C^{0}(\partial \Omega) \rightarrow C^{0}(\partial \Omega)$ is a certain boundary integral operator, which depends on the choice of the layer potential. 
Now we have to prove two conditions:

(i) The resulting operator $A_{n}$ is bounded and bijective.

(ii) $\int_{\partial \Omega}\left|D_{n}(x, y)\right| d o_{y} \leq c_{2}$ for all $x \in \mathbb{R}^{n}$.

The condition (i) implies that the inverse operator $A_{n}^{-1}$ is bounded, i.e. we have the estimate $\left\|A_{n}^{-1}\right\|_{\infty} \leq c_{1}$. Now using the estimate (ii) in addition, the asserted maximum modulus estimate follows from

$$
\begin{aligned}
& \max _{x \in \bar{\Omega}}|v(x)|= \\
& \max _{x \in \bar{\Omega}}\left|\int_{\partial \Omega} D_{n}(x, y) A_{n}^{-1} b(y) d o_{y}\right| \leq \\
& \max _{x \in \bar{\Omega}} \int_{\partial \Omega}\left|D_{n}(x, y)\right| \|\left. A_{n}^{-1}\right|_{\infty}|b(y)| d o_{y} \leq \\
& c_{1} c_{2} \max _{y \in \partial \Omega}|b(y)| .
\end{aligned}
$$

So the crucial point of this approach is to choose the layer potential representing the solution $v$ of the Stokes equation in such a way that both conditions (i) and (ii) are satisfied.

Let us start with a representation of the Stokes solution $v$ by a pure hydrodynamical double layer potential

$$
v(x):=D_{n} \psi(x):=\int_{\partial \Omega} D_{n}(x, y) \psi(y) d o_{y}, \quad x \in \Omega,
$$

where the $n \times n$ double layer kernel matrix

$$
D_{n}(x, y)=\left(D_{n}^{i, j}(x, y)\right)_{i, j=1, \ldots, n}
$$

is defined by

$$
D_{n}^{i, j}(x, y):=\frac{-2}{\omega_{n}} \frac{z_{i} z_{j} z \cdot N}{|z|^{n+2}} \quad z:=x-y, \quad \quad N:=N(y)
$$

with $\omega_{n}$ denoting the surface area of the $(n-1)$-dimensional unit sphere in $\mathbb{R}^{n}$.

Due to the jump relations of the double layer potential, when $x$ approaches the boundary, it turns out that the resulting Fredholm boundary integral operator has the form

$$
A_{n}:=\frac{1}{2} I_{n}+D_{n}
$$

where $I_{n}$ denotes the $n \times n$-identity matrix. It can be shown, however, that this boundary integral operator is not bijective, hence the condition (i) does not hold.

Therefore, instead of $A_{n}$, we use the boundary integral operator $\tilde{A}_{n}$, defined with help of a projection operator $P_{n}$ by

$$
\tilde{A}_{n}:=A_{n}-\beta P_{n}, \quad 0 \neq \beta \in \mathbb{R}, \quad P_{n} \psi(x):=\int_{\partial \Omega} \psi \cdot N \mathrm{~d} o N(x) .
$$


For the modified operator $\tilde{A}_{n}$ we can prove the following result.

Theorem 2.2: Let $2 \leq n \in \mathbb{N}$ and $0 \neq \beta \in \mathbb{R}$. Then it holds:

(a) The operator $\tilde{A}_{n}=\frac{1}{2} I_{n}+D_{n}-\beta P_{n}: C^{0}(\partial \Omega) \longrightarrow C^{0}(\partial \Omega)$ is bounded and bijective.

(b) Let $b \in C^{0}(\partial \Omega)$ be given satisfying the compatibility condition (1). Then the function

$$
\tilde{\psi}:=\tilde{A}_{n}^{-1} b \in C^{0}(\partial \Omega)
$$

is also a solution of the boundary integral equation $A_{n} \psi=b$ on $\partial \Omega$.

The above mentioned condition (ii) can also be proved, which implies the assertion of Theorem 2 , and hence the asserted maximum modulus estimate for the Stokes equations in bounded domains.

A maximum modulus estimate for the Stokes equations in unbounded exterior domains does also hold true. Here we can prove the following

Theorem 2.3: Let $\Omega \subset \mathbb{R}^{n}(n \geq 2)$ be an exteriore domain with a compact boundary $\partial \Omega \in C^{1, \alpha}$ $(\alpha>0)$. Let $b \in C^{0}(\partial \Omega)$ be given. Then there is a constant $c=c_{\Omega}>0$ such that the solution $v$ of the Stokes system satisfies a maximum modulus estimate of the type (2).

To prove condition (i) for the Stokes equation in unbounded domains, a representation of $v$ in form of a pure double layer potential is also not possible. Instead, we can use an ansatz in the form

$$
v(x):=D_{n} \psi(x)-\alpha E_{n} \psi(x):=D_{n} \psi(x)-\alpha \int_{\partial \Omega} E_{n}(x-y) \psi(y) d o_{y}, \quad x \in \Omega,
$$

where $D_{n} \psi$ is the above mentioned double layer potential and

$$
\begin{array}{rll}
E_{n} \Psi(x) & :=\int_{\partial \Omega} E_{n}(x-y) \Psi(y) d o_{y}, \\
E_{n}^{i, j}(x) & :=\frac{1}{2 \omega_{n}} \frac{x_{i} x_{j}}{|x|^{n}}+\frac{\delta_{i j}}{2 \omega_{n}} \begin{cases}-\ln |x| & , n=2 \\
(n-2)^{-1}|x|^{2-n} & , n \geq 3\end{cases}
\end{array}
$$

the corresponding single layer potential. The constant $\alpha$ must be chosen positive.

\section{References}

[1] R. Maremonti: On the maximum modulus theorem for the Stokes system. Ann. Scuola Normale SuperiorePisa, 21 (4) (1994), 629-643

[2] W. Kratz: The maximum modulus theorem for the Stokes system in a ball. Mathematische Zeitschrift, 226 (1997), 389-403

[3] W. Kratz: On the Maximum Modulus Theorem for Stokes Functions. Applicable Analysis, Vol. 58, 293-302

[4] W. Kratz: An extremal problem for Stokes functions in the plane. Analysis, no. 17 (1997), 219-225

[5] W. Varnhorn: The Stokes equations. Academie Verlag 1994 